\title{
Intradialytic Calcium Kinetics and Cardiovascular Disease in Chronic Hemodialysis Patients
}

\author{
Markus Pirklbauer ${ }^{\mathrm{a}}$ Lisa Fuchs $^{\mathrm{a}}$ Ramona Heiss $^{\mathrm{a}}$ Thomas Ratschiller ${ }^{\mathrm{b}}$ \\ Gert Mayer ${ }^{\mathrm{a}}$ \\ aDepartment of Internal Medicine IV - Nephrology and Hypertension, Medical University Innsbruck, \\ Innsbruck, Austria; 'bepartment of Cardiac, Vascular and Thoracic Surgery, Kepler University Hospital, \\ Linz, Austria
}

\section{Keywords \\ Calcium mass balance - Cardiovascular disease . \\ Exchangeable calcium pool · Hemodialysis · Osteocalcin}

\begin{abstract}
Background/Objective: Calcium loading has been associated with cardiovascular risk in hemodialysis (HD) patients. However, it remains to be elucidated whether alterations of intradialytic calcium buffering add to the increased cardiovascular disease burden in this high-risk population. Methods: Intradialytic calcium kinetics was evaluated in a crosssectional observational study by measuring dialysate-sided ionized calcium mass balance ( $\mathrm{iCa}_{\mathrm{MB}}$ ), calcium buffer capacity, and change in serum calcium levels in 40 chronic HD patients during a routine $\mathrm{HD}$ session. A dialysate calcium of 3.5 $\mathrm{mEq} / \mathrm{L}$ was used to adequately challenge calcium buffer mechanisms. Aortic pulse wave velocity and serum osteocalcin levels were measured prior to the HD session. Presence of cardiovascular disease and diabetes was assessed. $\boldsymbol{R e}$ sults: The mean dialysate-sided $\mathrm{iCa}_{\mathrm{MB}}$, extracellular fluid ionized calcium mass gain, and buffered ionized calcium mass were $469( \pm 154), 111( \pm 49)$, and $358( \pm 145) \mathrm{mg} / \mathrm{HD}$, respectively. The mean ionized serum calcium increase $(\Delta \mathrm{iCa})$ was
\end{abstract}

(C) 2020 The Author(s)

Published by S. Karger AG, Basel

This article is licensed under the Creative Commons AttributionNonCommercial-NoDerivatives 4.0 International License (CC BYNC-ND) (http://www.karger.com/Services/OpenAccessLicense). Usage and distribution for commercial purposes as well as any distribution of modified material requires written permission.
$0.42( \pm 0.14) \mathrm{mEq} / \mathrm{L}$ per HD. The mean intradialytic calcium buffer capacity was $73( \pm 18) \%$. Multivariate regression analysis revealed significant independent association of (1) iCa $\mathrm{Ca}_{M B}$ with the dialysate-to-blood calcium gradient at HD start and (2) intradialytic calcium buffer capacity with undercarboxylated osteocalcin. The presence of coronary heart disease was associated with higher $\triangle \mathrm{iCa}$ but not $\mathrm{iCa}_{\mathrm{MB}}$ in the multivariate model. Conclusions: In line with our proof-of-concept study, we provide clinical evidence for a rapidly accessible and exchangeable calcium pool involved in intradialytic calcium regulation and for the role of osteocalcin as a potential biomarker. Our findings argue for evaluating the prognostic potential of intradialytic calcium kinetics in prospective clinical trials.

(c) 2020 The Author(s)

Published by S. Karger AG, Basel

\section{Introduction}

Current KDIGO guidelines [1] recommend a dialysate calcium $(\mathrm{dCa})$ concentration of $2.5-3 \mathrm{mEq} / \mathrm{L}$ in order to avoid excessive intradialytic calcium (Ca) loading as this has been associated with an elevated cardiovascular (CV) risk [2]. However, we and others have previously demon- 
strated that even under these precautions calcium loading during dialysis occurs in a relevant number of chronic hemodialysis (HD) patients. For example, Gotch et al. [3] calculated that up to $40 \%$ of chronic HD patients are still in a positive overall $\mathrm{Ca}$ mass balance $\left(\mathrm{Ca}_{\mathrm{MB}}\right)$ with a $\mathrm{dCa}$ concentration of $2.5 \mathrm{mEq} / \mathrm{L}$ when considering both interand intradialytic balance. However, even without taking interdialytic calcium uptake into account, a substantial interindividual variability of intradialytic $\mathrm{Ca}_{\mathrm{MB}}$ ranging from -500 up to $+400 \mathrm{mg} / \mathrm{HD}$ session has been observed with the use of $2.5 \mathrm{mEq} / \mathrm{L} \mathrm{dCa}$ [4-6]. As the main determinant for calcium transfer during HD is the predialysis serum calcium concentration, these differences are most likely due to the varying use of active vitamin $\mathrm{D}$ and/or calcimimetic drugs but different $\mathrm{Ca}_{\mathrm{MB}}$ assessment approaches have also to be taken into consideration $[7,8]$. By utilizing a dialysate-sided ionized $\mathrm{Ca}_{\mathrm{MB}}\left(\mathrm{iCa}_{\mathrm{MB}}\right)$ assessment approach, we previously demonstrated that the use of both 2.5 and $3.5 \mathrm{mEq} / \mathrm{L}$ dCa regularly results in a highly positive intradialytic $\mathrm{iCa}_{\mathrm{MB}}$, which is primarily predicted by the predialysis dialysate-to-blood ionized $\mathrm{Ca}$ gradient [4]. The $\mathrm{Ca}_{\mathrm{MB}}$ was not reflected by changes in serum Ca concentration corroborating previous findings that the latter is not a reliable marker of Ca burden [9]. This is because a rapidly accessible and exchangeable $\mathrm{Ca}$ pool counteracts fluctuations of serum Ca deviations on a minute-to-minute basis in the event of high intradialytic Ca loading independent of calcitropic hormones, intestinal and renal Ca handling as well as cellular bone remodeling. This concept has been previously postulated [10] and corroborated for the first time in vivo in our clinical proof-of-concept study by reporting individual Ca buffer capacity in 28 chronic HD patients, ranging from 60 to $100 \%$ of whole individual intradialytic Ca load [4]. The lack of sensitivity of changes in serum Ca concentration and differences in the individual ability to remove excess $\mathrm{Ca}$ from the extracellular fluid (ECF) compartment implicate that dialysate-sided $\mathrm{iCa}_{\mathrm{MB}}$ assessment is less error-prone compared with blood-sampling based $\mathrm{Ca}_{\mathrm{MB}}$ assessment strategies but rather requires partial or complete dialysate fluid collection.

If indeed an altered intradialytic Ca buffering has clinical consequences, such as a higher associated CV risk, is not completely clear. For example, Yamada et al. [11] previously demonstrated that intradialytic change in serum total $\mathrm{Ca}(\Delta \mathrm{tCa})$ is associated with aortic calcification in HD patients and Tagawa et al. [12] recently showed that higher $\mathrm{dCa}$ is associated with myocardial infarction among diabetic HD patients, which was statistically explained through an effect on $\Delta \mathrm{tCa}$. However, in the ab- sence of $\mathrm{Ca}_{\mathrm{MB}}$ assessment in both studies, it remains to be elucidated whether high intradialytic Ca loading and/or impaired Ca buffering per se increase CV risk. We, thus, conducted a cross-sectional, observational clinical study assessing intradialytic Ca kinetics (i.e., dialysate-sided $\mathrm{iCa}_{\mathrm{MB}}$, intradialytic Ca buffer capacity, and change in ionized and total serum Ca levels) and its association with the prevalence of $\mathrm{CV}$ disease in 40 chronic $\mathrm{HD}$ patients.

\section{Materials and Methods}

\section{Study Population and Procedures}

Fourty chronic end-stage renal disease patients who underwent bicarbonate HD for more than 1 month were recruited to undergo noninvasive assessment of intradialytic $\mathrm{Ca}$ kinetics (i.e., dialysatesided $\mathrm{iCa}_{\mathrm{MB}}$, intradialytic Ca buffer capacity, and change in serum Ca levels; see definitions below), serum osteocalcin measurement as well as assessment of aortic pulse wave velocity (PWV) and blood- and pulse-pressure during a regular HD session. Exclusion criteria were inability or unwillingness to provide written informed consent, age below 18 years, and predialysis serum ionized $\mathrm{Ca}$ (iCa) concentration above $2.7 \mathrm{mEq} / \mathrm{L}$; however, no patient was excluded based on these a priori established criteria. Study patients were not part of our previous proof-of-concept study [4]. HD treatment followed patients' routine prescriptions with a dCa set to $3.5 \mathrm{mEq} / \mathrm{L}$ for a single $\mathrm{HD}$ session in order to adequately challenge the intradialytic Ca buffer mechanisms. Aiming at a constant ultrafiltration during the session, only mid- and end of the week HD sessions were used for study procedures. The vascular access was evaluated by measuring recirculation using the dialysis device (Fresenius 5008). Only subjects with recirculation values below $10 \%$ were qualified for study participation.

\section{Blood-and Dialysate-Based Measurements}

To assess intradialytic Ca kinetics, blood was drawn before the dialyzer (i.e., "precapillary") prior to the beginning and then every 30 min until the end of each HD session to determine the respective precapillary iCa concentrations. In parallel, iCa concentrations were determined after 15, 30, 45, $60 \mathrm{~min}$, and then every 30 min until the end of the dialysis session both in the fresh and spent dialysate. Ionized $\mathrm{dCa}$ in fresh and spent dialysate as well as precapillary serum iCa concentrations were analyzed by using an onsite blood gas analysis device (ABL 800 Flex, Drott Medizintechnik $\mathrm{GmbH}$, Wiener Neudorf, Austria) with the appropriate settings for dialysate and serum specimen according to the manufacturer's instructions. In addition, serum samples were collected before the $\mathrm{HD}$ session in each study participant and stored at $-80^{\circ} \mathrm{C}$ for ELISA-based osteocalcin analysis. Serum carboxylated osteocalcin (cOC) and undercarboxylated osteocalcin (ucOC) were measured using respective high-sensitive enzyme immunoassay kits (Takara Bio Europe/Clontech, Saint-Germain-en-Laye, France) according to the manufacturer's instructions. Serum total $\mathrm{Ca}(\mathrm{tCa})$ was measured using a colorimetric NM-BAPTA chromophore-based assay (Roche Diagnostics GmBH, Mannheim, Germany) by the Central Institute for Medical and Chemical Laboratory Diagnostics (ZIMCL) at the Medical University Innsbruck/University Hospital Innsbruck. 


\section{Assessment of Dialysate-Sided $i C a_{M B}$}

$\mathrm{iCa}_{\mathrm{MB}}$ was assessed according to a previously published approach $[4,5,13]$, which estimates net Ca mass differences between the inlet and outlet dialysate tubing for each 30-min HD session time period and includes both the diffusive and convective component of $\mathrm{iCa}_{\mathrm{MB}}$ :

$$
\mathrm{iCa}_{\mathrm{MB}}=\mathrm{dCa}_{\text {mean in }} \times Q_{\mathrm{D}} \times t-\sum_{k=0}^{9}\left\{\left(k \times Q_{\mathrm{D}} \times 30\right)+(k \times \mathrm{UF} \times 30)\right\},
$$

where $\mathrm{iCa}_{\mathrm{MB}}(\mathrm{mEq} / \mathrm{HD})$ is the ionized calcium mass balance; $\mathrm{dCa}$ mean in $(\mathrm{mEq} / \mathrm{L})$ is the mean precapillary dCa over the dialysis session; $Q_{\mathrm{D}}(\mathrm{L} / \mathrm{min})$ is the dialysate flow rate; $t(\mathrm{~min})$ is the duration of dialysis session; $k$ is the postcapillary $\mathrm{dCa}(\mathrm{mEq} / \mathrm{L})$ measured at $0 \min (k=1), 30 \min (k=2), 60 \min (k=3), 90 \min (k=$ 4), $120 \mathrm{~min}(k=5), 150 \mathrm{~min}(k=6), 180 \mathrm{~min}(k=7), 210 \mathrm{~min}(k=$ $8)$, and $240 \mathrm{~min}(k=9)$; UF $(\mathrm{L} / \mathrm{min})$ is the ultrafiltration rate; and $\mathrm{Ca}_{\mathrm{MB}}(\mathrm{mg} / \mathrm{HD})=\mathrm{Ca}_{\mathrm{MB}}$ in $\mathrm{mEq} / \mathrm{HD} \times 80.16$.

\section{Calculation of Intradialytic Calcium Buffer Capacity}

Intradialytic $\mathrm{Ca}$ buffer capacity was determined by setting the intradialytic change in ECF ionized Ca mass $\left(\triangle \mathrm{iCa} a_{\mathrm{ECF}}\right)$ in relation to $\mathrm{iCa}_{\mathrm{MB}}$ :

Intradialytic calcium buffer capacity ( $\%$ of whole $\mathrm{Ca}$ load/HD session $)=\left(1-\Delta \mathrm{iCA}_{\mathrm{ECF}} / \mathrm{iCA}_{\mathrm{MB}}\right) \times 100$

The intradialytic increase in serum ionized $\mathrm{Ca}(\mathrm{iCa})$ concentration was used for calculation of $\triangle \mathrm{iCa}_{\mathrm{ECF}}(\mathrm{mg} / \mathrm{HD})$. Similar iCa kinetics in ECF and serum was assumed according to Andersen et al. who showed only a small difference (approx. 5\%) between serum and interstitial fluid iCa concentrations [14]:

$$
\Delta \mathrm{iCa}_{\mathrm{ECF}}(\mathrm{mg} / \mathrm{HD})=\text { post-HD iCa }(\mathrm{mg} / \mathrm{L}) \times
$$
post-HD ECF $(\mathrm{L})-$ pre-HD iCa $(\mathrm{mg} / \mathrm{L}) \times$ pre-HD ECF $(\mathrm{L})$

ECF volume was calculated at the beginning and end of the HD session based on patient weight before and after the HD session as $20 \%$ of patient weight before and after the HD session, respectively, to account for ultrafiltration-based ECF volume changes. Of note, the cellular compartment does not add to the distribution volume of $\mathrm{iCa}$ as an extremely low intracellular Ca concentration is mandatory for electrophysiologic reason.

Calculation of Intradialytic Change in Serum Ca Levels

The intradialytic change in serum $\mathrm{iCa}(\Delta \mathrm{iCa})$ and $\mathrm{tCa}(\Delta \mathrm{tCa})$ levels was calculated as follows:

$$
\begin{aligned}
& \Delta \mathrm{iCa}(\mathrm{mEq} / \mathrm{L})=\mathrm{iCa} \text { post-HD }(\mathrm{mEq} / \mathrm{L})-\mathrm{iCa} \text { pre-HD } \\
& (\mathrm{mEq} / \mathrm{L}) \\
& \quad \Delta \mathrm{tCa}(\mathrm{mEq} / \mathrm{L})=\mathrm{tCa} \text { post-HD }(\mathrm{mEq} / \mathrm{L})-\mathrm{tCa} \text { pre-HD } \\
& (\mathrm{mEq} / \mathrm{L})
\end{aligned}
$$

\section{Data Collection for Cross-Sectional Analysis}

Clinical parameters of vascular calcification, namely, aortic PWV and peripheral blood and pulse pressure, were measured noninvasively before the midweek dialysis session in a calm environment with the patient in supine position after 5 min of supine rest. Brachial systolic blood pressure (SBP) and diastolic blood pressure (DBP) were calculated as the mean values of a minimum of 3 measurements taken at least $3 \mathrm{~min}$ apart on the nonfistula arm using a validated sphygmomanometer (HEM-780-D, Omron, Kyoto, Japan). Aortic PWV was measured by an oscillometric method (Mobil-O-Graph 24 h PWA device, IEM GmbH, Stolberg, Germany) utilizing an ARCsolver algorithm-based pulse wave analysis [15].

Ultrafiltration rate, dialysate flow rate, effective blood flow, and duration of dialysis session were obtained from the HD device (Fresenius 5008). Medical treatment (type and quantity of phosphate binders used, type and quantity of vitamin D substitution, and quantity of calcimimetic drugs) over the last 6 months or since dialysis onset in case of more recent dialysis start, comorbidities (diabetes status, smoking status, coronary heart disease, congestive heart failure, and peripheral arterial occlusive disease), demographic parameters (e.g., age and gender), and routinely collected laboratory parameters (e.g., hemoglobin, hematocrit, $\mathrm{pH}$, serum bicarbonate, serum albumin, serum protein, serum phosphate, serum $\mathrm{Ca}$, and serum PTH levels) were obtained from our local computerized clinical documentation systems and documented using a standardized case report form.

\section{Definition of Comorbidities and Clinical Events}

Coronary heart disease (CHD)/coronary artery disease $=$ history of myocardial infarction and/or coronary artery bypass graft and/or coronary artery angioplasty.

Peripheral arterial occlusive disease $(\mathrm{PAOD})=$ history of documented Fontaine Stage II, III, or IV.

Congestive heart failure $(\mathrm{CHF})=$ history of hospital admission due to nonpneumopathological dyspnea and/or acute cardiogenic pulmonary edema documented on chest X-ray and left ventricular ejection fraction (LVEF) $<40 \%$ as documented by echocardiography.

Diabetes mellitus $(\mathrm{DM})=$ history of diabetes type I or II and use of a hypoglycemic agent or insulin.

\section{Statistical Analysis}

A priori sample size calculation was based on the results of our previous proof-of-concept study in $28 \mathrm{HD}$ patients [4]: with a reported mean intradialytic calcium buffer capacity of $78( \pm 7) \%, 40$ patients would allow for a $95 \%$ CI of $\pm 2.2 \%$. The results of intradialytic Ca kinetics assessment and baseline characteristics are presented as mean values $( \pm S D)$ or absolute frequencies. Associations among intradialytic $\mathrm{Ca}$ kinetics parameters and osteocalcin levels, CV disease, comorbidities, laboratory and demographic parameters, and medical treatment as well as clinical parameters of vascular calcification (PWV and peripheral blood and pulse pressure) were assessed by means of correlation and regression analysis. Parametric distribution of the data was assessed by the use of Kolmogorov-Smirnov test. Pearson's correlation coefficient $(r)$ or Kendall's Tau-b coefficient were computed for parametric and nonparametric correlation of metric variables, respectively. The nonparametric Mann-Whitney $U$ test was used to compare the distribution of metric variables among dichotomized nominal parameters. To identify independent predictors of intradialytic $\mathrm{Ca}$ kinetics and CV disease, only parameters that showed a significant bivariate correlation (metric variables) or a significantly different distribution (nominal variables) were entered into a multiple linear (metric variables) or logistic (nominal variables) regression model, respectively. The results are presented as standardized beta coefficients $(\beta)$ or regression coefficient B, respectively. Statistical analysis was performed with SPSS version 24.0. The level of significance ( $p$ value) was set to 0.05 . Adjustment for multiple testing was not performed. 
Table 1. Patient cohort characteristics $(n=40)$

\begin{tabular}{|c|c|c|}
\hline & Mean & SD \\
\hline \multicolumn{3}{|l|}{ Demographics } \\
\hline Age, years & 62.05 & 14.29 \\
\hline Dialysis vintage, years & 1.67 & 2.82 \\
\hline \multicolumn{3}{|l|}{ Laboratory parameters } \\
\hline Serum phosphate, $\mathrm{mg} / \mathrm{dL}^{\mathrm{a}}$ & 4.6 & 0.87 \\
\hline Serum total $\mathrm{Ca}, \mathrm{mEq} / \mathrm{L}^{\mathrm{a}}$ & 4.38 & 0.26 \\
\hline Ionized serum $\mathrm{Ca}$ at $\mathrm{HD}$ start, $\mathrm{mEq} / \mathrm{L}$ & 2.32 & 0.12 \\
\hline Ionized serum $\mathrm{Ca}$ at $\mathrm{HD}$ end, $\mathrm{mEq} / \mathrm{L}^{\mathrm{b}}$ & 2.74 & 0.1 \\
\hline Total serum $\mathrm{Ca}$ at $\mathrm{HD}$ start, $\mathrm{mEq} / \mathrm{L}$ & 4.5 & 0.26 \\
\hline Total serum $\mathrm{Ca}$ at $\mathrm{HD}$ end, $\mathrm{mEq} / \mathrm{L}^{\mathrm{b}}$ & 5.4 & 0.26 \\
\hline Serum albumin, mg/dL & $3,558.50$ & 466.05 \\
\hline Serum protein, $\mathrm{g} / \mathrm{dL}$ & 6.93 & 0.62 \\
\hline Serum PTH, $\mathrm{pg} / \mathrm{mL}^{\mathrm{a}}$ & 307.31 & 133.95 \\
\hline Serum PTH pre HD, pg/mL & 294.38 & 218.24 \\
\hline Serum PTH post $\mathrm{HD}, \mathrm{pg} / \mathrm{mL}^{\mathrm{b}}$ & 59.31 & 41.59 \\
\hline Serum alkaline phosphatase $\left(\mathrm{U} / \mathrm{L}^{\mathrm{a}}\right.$ & 105.26 & 59.97 \\
\hline Serum LDL cholesterol, mg/dL & 93.44 & 49.48 \\
\hline Serum HDL cholesterol, mg/dL & 48.59 & 23.22 \\
\hline Serum LDL/HDL cholesterol ratio ${ }^{\mathrm{a}}$ & 1.96 & 1.20 \\
\hline Hemoglobin, $\mathrm{g} / \mathrm{dL}^{\mathrm{a}}$ & 10.64 & 0.65 \\
\hline Hemoglobin pre $\mathrm{HD}, \mathrm{g} / \mathrm{dL}$ & 11.36 & 1.00 \\
\hline Hemoglobin post $\mathrm{HD}, \mathrm{g} / \mathrm{dL}^{\mathrm{b}}$ & 11.89 & 1.38 \\
\hline Serum hematocrit pre HD, \% & 35.02 & 3.04 \\
\hline Serum hematocrit post $\mathrm{HD}, \%^{\mathrm{b}}$ & 36.60 & 4.13 \\
\hline Serum C-reactive protein $(\mathrm{mg} / \mathrm{dL})$ & 0.88 & 0.96 \\
\hline Serum $\left[\mathrm{HCO}_{3}^{-}\right]$pre $\mathrm{HD}, \mathrm{mEq} / \mathrm{L}$ & 21.25 & 1.87 \\
\hline Serum $\left[\mathrm{HCO}_{3}^{-}\right]$post $\mathrm{HD}, \mathrm{mEq} / \mathrm{L}^{\mathrm{b}}$ & 25.68 & 1.88 \\
\hline Serum $\mathrm{pH}$ pre HD & 7.37 & 0.05 \\
\hline Serum $\mathrm{pH}$ post $\mathrm{HD}^{\mathrm{b}}$ & 7.47 & 0.04 \\
\hline Serum cOC, ng/mL & 50.53 & 34.4 \\
\hline Serum ucOC, ng/mL & 95.00 & 111.05 \\
\hline \multicolumn{3}{|l|}{ Physical examination } \\
\hline Dry weight, kg & 74.48 & 16.49 \\
\hline Height, $\mathrm{cm}$ & 171.62 & 8.46 \\
\hline Body mass index, $\mathrm{kg} / \mathrm{m}^{2}$ & 25.51 & 5.42 \\
\hline Systolic RR, mm Hg & 146.67 & 14.88 \\
\hline Diastolic RR, mm Hg & 72.09 & 15.17 \\
\hline Pulse pressure, mm Hg & 74.87 & 16.68 \\
\hline Pulse wave velocity, $\mathrm{m} / \mathrm{s}$ & 9.40 & 1.97 \\
\hline \multicolumn{3}{|l|}{ Dialysis-specific parameters } \\
\hline Ultrafiltration rate, $\mathrm{mL} / \mathrm{HD}$ & $1,791.68$ & $1,114.64$ \\
\hline HD session lenght, min & 236.25 & 13.90 \\
\hline Dialysate flow rate, $\mathrm{mL} / \mathrm{min}$ & 786.75 & 88.37 \\
\hline Dialysate-to-blood iCa gradient at $\mathrm{HD}$ start, $\mathrm{mEq} / \mathrm{L}$ & 0.60 & 0.16 \\
\hline Dialysate $\left[\mathrm{HCO}_{3}^{-}\right], \mathrm{mEq} / \mathrm{L}$ & 29.5 & 1.8 \\
\hline Measured ionized prefilter $\mathrm{dCa}, \mathrm{mEq} / \mathrm{L}$ & 2.92 & 0.12 \\
\hline Calcium containing phosphate binder dose, $\mathrm{g} / \mathrm{day}^{\mathrm{a}}$ & 1.10 & 1.34 \\
\hline Non-calcium containing phosphate binder dose, $\mathrm{g} / \mathrm{day}^{\mathrm{a}}$ & 4.23 & 2.29 \\
\hline Active vitamin $\mathrm{d}$ dose, $\mathrm{yg} / \mathrm{week}^{\mathrm{a}}$ & 1.19 & 1.50 \\
\hline
\end{tabular}


Table 1 (continued)

\begin{tabular}{ll}
\hline & $\%$ \\
\hline Sex & 33 (female)/67 (male) \\
Comorbidities & Yes/no \\
$\quad$ Ever smoker & $31 / 69$ \\
Current smoker & $13 / 87$ \\
DM & $43 / 57$ \\
CAD & $33 / 67$ \\
CHF & $13 / 87$ \\
PAOD & $28 / 72$ \\
Medication & Yes/no \\
Cinacalcet use & $15 / 85$ \\
Vitamin Kantagonist use & $15 / 85$ \\
\hline
\end{tabular}

PWV, pulse wave velocity; cOC, carboxylated osteocalcin; ucOC, undercarboxylated osteocalcin; iCa, ionized $\mathrm{Ca}$; $\mathrm{dCa}$, dialysate calcium; $\mathrm{CAD}$, coronary artery disease; $\mathrm{CHF}$, congestive heart failure; $\mathrm{PAOD}$, peripheral arterial occlusive disease; DM, diabetes mellitus. ${ }^{\text {a }}$ Indicating an average value over the last 6 months or since dialysis onset in case of more recent dialysis start. Conversion factors for units: total calcium in $\mathrm{mg} / \mathrm{dL}$ to $\mathrm{mmol} / \mathrm{L}$, $\times 0.2495$; ionized calcium in $\mathrm{mEq} / \mathrm{L}$ to $\mathrm{mmol} / \mathrm{L}, \times 0.5$; LDL cholesterol in $\mathrm{mg} / \mathrm{dL}$ to $\mathrm{mmol} / \mathrm{L}, \times 0.02586$; HDL cholesterol in $\mathrm{mg} / \mathrm{dL}$ to $\mathrm{mmol} / \mathrm{L}, \times 0.02586$; phosphorus in $\mathrm{mg} / \mathrm{dL}$ to $\mathrm{mmol} / \mathrm{L}, \times 0.3229 .{ }^{\mathrm{b}}$ Significantly different from HD start $(p<0.001$, paired $t$ test). Carboxylated (cOC) and undercarboxylated (ucOC) osteocalcin.

\section{Results}

Intradialytic Ca kinetics was assessed in 40 chronic $\mathrm{HD}$ patients during a routine dialysis session (see Table 1 for patient cohort characteristics). Using $3.5 \mathrm{mEq} / \mathrm{L} \mathrm{dCa}$ allowed to adequately challenge intradialytic Ca buffer mechanisms and led to a positive $\mathrm{iCa}_{\mathrm{MB}}$ in all patients with a Ca load of $469( \pm 154) \mathrm{mg} / \mathrm{HD}$ on average. The mean intradialytic $\Delta \mathrm{iCa}$ and $\Delta \mathrm{tCa}$ was $0.42( \pm 0.14)$ and $0.9( \pm 0.32) \mathrm{mEq} / \mathrm{L}$, respectively (Table 2$)$. With a calculated mean $\triangle \mathrm{iCa}_{\mathrm{ECF}}$ of $111( \pm 49) \mathrm{mg} / \mathrm{HD}$, the averaged amount of buffered iCa mass was $358( \pm 145) \mathrm{mg} / \mathrm{HD}$. Thus, the mean intradialytic iCa buffer capacity (i.e., relation between $\triangle \mathrm{iCa}_{\mathrm{ECF}}$ and $\left.\mathrm{iCa}_{\mathrm{MB}}\right)$ was $73( \pm 18) \%$. In order to identify predictors of intradialytic Ca kinetics parameters, univariate correlation analysis was conducted: $\mathrm{iCa}_{\mathrm{MB}}$ correlated positively with the dialysate-to-blood iCa gradient at HD start $(p<0.05)$ and negatively with serum bicarbonate at HD start and mean hemoglobin (Hb) levels ( $p<0.01$ each). For intradialytic Ca buffer capacity, we found a positive correlation with serum ucOC $(p<0.05)$ and a negative correlation with dry weight $(p<$ $0.01) . \triangle \mathrm{iCa}$ was positively associated with the dialysateto-blood iCa gradient at HD start $(p<0.01)$ and negatively associated with serum bicarbonate at HD end ( $p<$ 0.05) (Table 3).

These predictors of intradialytic calcium kinetics were entered in a multiple linear regression model: Here, we
Table 2. Intradialytic calcium kinetic parameters $(n=40)$

\begin{tabular}{lrr}
\hline & $\begin{array}{l}\text { Mean } \\
(\mathrm{SD})\end{array}$ & \multicolumn{1}{c}{$\mathrm{SD}$} \\
\hline iCa mass balance, mg/HD & 469 & 154 \\
Intradialytic ECF iCa mass gain, mg/HD & 111 & 49 \\
Buffered iCa mass, mg/HD & 358 & 145 \\
Intradialytic Ca buffer capacity (\% of load/HD) & 73 & 18 \\
$\Delta \mathrm{iCa}, \mathrm{mEq} / \mathrm{L}^{\mathrm{a}}$ & 0.42 & 0.14 \\
$\Delta \mathrm{tCa}, \mathrm{mEq} / \mathrm{L}^{\mathrm{a}}$ & 0.9 & 0.32 \\
\hline
\end{tabular}

$\mathrm{iCa}$, ionized $\mathrm{Ca} ; \Delta \mathrm{iCa}$, ionized serum calcium increase; $\Delta \mathrm{tCa}$, serum total Ca; HD, hemodialysis; ECF, extracellular fluid. a Statistically significant change $(\Delta)$ from HD start to HD end $(p<$ 0.001 , paired $t$ test).

could demonstrate an independent association of $\mathrm{iCa}_{\mathrm{MB}}$ with measured dialysate-to-blood iCa gradient at $\mathrm{HD}$ start $(\beta=0.341$ and $p=0.01)$, that is, a higher dialysateto-blood iCa gradient relates to higher $\mathrm{iCa}_{\mathrm{MB}}$. In addition, $\mathrm{iCa}_{\mathrm{MB}}$ was independently associated with serum bicarbonate at HD start $(\beta=-0.361$ and $p=0.007)$ and mean $\mathrm{Hb}$ levels $(\beta=0.439$ and $p=0.001)$. Intradialytic Ca buffer capacity was found to be independently associated with ucOC $(\beta=0.461$ and $p=0.004)$ and dry weight $(\beta=$ -0.398 and $p=0.011) . \Delta \mathrm{iCa}$ was independently associated with the dialysate-to-blood iCa gradient at dialysis start only $(\beta=0.772$ and $p<0.001)$ (Table 4$)$. 
Table 3. Bivariate correlation coefficients (Pearson $r$ or Kendall's Tau-b) of $\mathrm{iCa}_{\mathrm{MB}}$, intradialytic Ca buffer capacity, $\triangle \mathrm{iCa}$, and PWV with continuous variables

\begin{tabular}{|c|c|c|c|}
\hline Continuous variables & $r$ & $b$ & $p$ value \\
\hline \multicolumn{4}{|l|}{$\mathrm{iCa}_{\mathrm{MB}}, \mathrm{mg} / \mathrm{HD}$} \\
\hline Dialysate-to-blood iCa gradient at HD start, mEq/L & 0.356 & & $<0.05$ \\
\hline $\mathrm{Hb}, \mathrm{g} / \mathrm{dL}^{\mathrm{a}}$ & 0.405 & & $<0.01$ \\
\hline Serum $\mathrm{HCO}_{3}{ }^{-}$at $\mathrm{HD}$ start, $\mathrm{mEq} / \mathrm{L}$ & -0.411 & & $<0.01$ \\
\hline \multicolumn{4}{|l|}{ Intradialytic calcium buffer capacity, \% of load/HD } \\
\hline Dry weight, $\mathrm{kg}$ & -0.340 & & $<0.01$ \\
\hline Serum ucOC, ng/mL & & 0.281 & $<0.05$ \\
\hline \multicolumn{4}{|l|}{$\Delta \mathrm{iCa}, \mathrm{mEq} / \mathrm{L}$} \\
\hline Dialysate-to-blood iCa gradient at HD start, mEq/L & 0.771 & & $<0.01$ \\
\hline $\begin{array}{l}\text { Serum } \mathrm{HCO}_{3}{ }^{-} \text {at } \mathrm{HD} \text { end, } \mathrm{mEq} / \mathrm{L} \\
\mathrm{PWV}, \mathrm{m} / \mathrm{s}\end{array}$ & -0.391 & & $<0.05$ \\
\hline Age, years & 0.917 & & $<0.001$ \\
\hline Serum cOC, ng/mL & & -0.323 & $<0.05$ \\
\hline C-reactive protein, $\mathrm{mg} / \mathrm{dL}$ & & 0.297 & $<0.05$ \\
\hline Dialysis vintage, days & & -0.275 & $<0.05$ \\
\hline
\end{tabular}

$\mathrm{iCa}_{\mathrm{MB}}$, ionized calcium mass balance; PWV, pulse wave velocity; HD, hemodialysis; $\mathrm{iCa}$, ionized $\mathrm{Ca} ; \mathrm{iCa}$, ionized serum calcium increase; cOC, carboxylated osteocalcin. ${ }^{a}$ Indicating an average value over the last 6 months or since dialysis onset in case of more recent dialysis start.

Table 4. Multiple linear regression analysis for $\mathrm{iCa}_{\mathrm{MB}}$, intradialytic Ca buffer capacity, $\Delta \mathrm{iCa}$, and $\mathrm{PWV}$ as dependent variables

\begin{tabular}{|c|c|c|c|}
\hline Dependent variable & Independent variables & $\beta$ & $p$ value \\
\hline \multirow[t]{2}{*}{$\mathrm{iCa}_{\mathrm{MB}}, \mathrm{mg} / \mathrm{HD}$} & Dialysate-to-blood iCa gradient at HD start, mEg/L & 0.341 & 0.01 \\
\hline & $\mathrm{Hb}, \mathrm{g} / \mathrm{dL}^{\mathrm{a}}$ & 0.439 & 0.001 \\
\hline \multirow[t]{2}{*}{ Intradialytic Ca buffer capacity, $\%$ of load/HD } & Serum ucOC, ng/mL & 0.461 & 0.004 \\
\hline & Dry weight, kg & -0.398 & 0.011 \\
\hline \multirow{2}{*}{$\Delta \mathrm{iCa}, \mathrm{mEq} / \mathrm{L}$} & Start, $\mathrm{mEq} / \mathrm{L}$ & & \\
\hline & Serum $\mathrm{HCO}_{3}{ }^{-}$at $\mathrm{HD}$ end, $\mathrm{mEq} / \mathrm{L}$ & 0.007 & 0.957 \\
\hline \multirow[t]{3}{*}{$\mathrm{PWV}, \mathrm{m} / \mathrm{s}$} & Age & 0.894 & $<0.001$ \\
\hline & Serum cOC, ng/mL & -0.064 & 0.478 \\
\hline & C-reactive protein, $\mathrm{mg} / \mathrm{dL}$ & 0.118 & 0.178 \\
\hline
\end{tabular}

$\mathrm{iCa}_{\mathrm{MB}}$, ionized calcium mass balance; $\mathrm{HD}$, hemodialysis; iCa, ionized $\mathrm{Ca}$; $\mathrm{COC}$, carboxylated osteocalcin. Results are presented as standardized beta coefficient $(\beta)$ and $p$ value for significance of difference. ${ }^{\text {a }}$ Indicating an average value over the last 6 months or since dialysis onset in case of more recent dialysis start. ${ }^{b}$ Only values for intradialytic Ca buffer capacity $>60 \%$ (i.e., $83 \%$ of patients) were included in the linear regression model (i.e., 7 outliers were excluded).

We next aimed at identifying potential associations between calcium kinetics parameters and $\mathrm{CV}$ endpoints (CHD, PAOD, CHF, and CVD) as well as DM status by applying univariate correlation analysis: The presence of $\mathrm{CHD}$ was associated with higher $\mathrm{iCa}_{\mathrm{MB}}$ and higher $\Delta \mathrm{iCa}$ $(p<0.05$ each). The presence of DM was associated with lower intradialytic Ca buffer capacity $(p<0.01)$ (Table 5). Aortic PWV, an established clinical parameter of vascular calcification, correlated negatively with serum $\operatorname{cOC}(p<$ $0.05)$ (Table 3). Multiple logistic regression analysis showed an independent positive association of $\Delta \mathrm{iCa}$ with the presence of $\mathrm{CHD}(B=12.8$ and $p=0.04)$; however, this did not hold true for $\mathrm{iCa}_{\mathrm{MB}}$ (Table 6). No further significant associations were found between calcium kinetics parameters, $\mathrm{CV}$ endpoints, and any other reported patients characteristics (Table 1). 
Table 5. Comparison of distribution across dichotomous nominal variables (coronary heart disease and DM status) using Mann-Whitney $U$ test ( $U$ and $Z$ statistics)

\begin{tabular}{|c|c|c|c|}
\hline Continuous variables & $U$ & $Z$ & $p$ value \\
\hline \multicolumn{4}{|l|}{ CHD (yes/no) } \\
\hline$\Delta \mathrm{iCa}, \mathrm{mEq} / \mathrm{L}^{\mathrm{a}}$ & 94.5 & -2.35 & $<0.05$ \\
\hline $\mathrm{iCa}_{\mathrm{MB}}, \mathrm{mg} / \mathrm{HD}^{\mathrm{a}}$ & 90 & -2.47 & $<0.05$ \\
\hline \multicolumn{4}{|l|}{ DM status (yes/no) } \\
\hline Intradialytic calcium buffer capacity, $\%$ of load/HD & 95 & -2.75 & $<0.01$ \\
\hline Vintage, days ${ }^{\mathrm{b}}$ & 117 & -2.15 & $<0.05$ \\
\hline Pulse pressure, $\mathrm{mm} \mathrm{Hg}^{\mathrm{c}}$ & 108 & -2.39 & $<0.05$ \\
\hline
\end{tabular}

Table 6. Multiple logistic regression analysis for dichotomous nominal variables (coronary heart disease and DM status) as dependent variables

\begin{tabular}{llrr}
\hline Dependent nominal variable & Independent variables & $\beta$ & $p$ value \\
\hline CHD (yes/no) & $\Delta \mathrm{iCa}, \mathrm{mEq} / \mathrm{L}$ & 12.788 & 0.044 \\
& $\mathrm{iCa}_{\mathrm{MB}}, \mathrm{mg} / \mathrm{HD}$ & 0.005 & 0.068 \\
\hline \multirow{2}{*}{ DM status (yes/no) } & Pulse pressure, mm Hg & 0.055 & 0.037 \\
& Intradialytic calcium buffer capacity, \% of load/HD & -0.036 & 0.099 \\
& Vintage, days & -0.001 & 0.205 \\
\hline
\end{tabular}

$\mathrm{DM}$, diabetes mellitus; $\mathrm{iCa}_{\mathrm{MB}}$, ionized calcium mass balance; $\mathrm{HD}$, hemodialysis; $\mathrm{CHD}$, coronary heart disease. Results are presented as beta coefficient $(\beta)$ and $p$ value for significance of difference.

\section{Discussion}

The present cross-sectional clinical study assessed intradialytic $\mathrm{Ca}$ kinetics (i.e., dialysate-sided $\mathrm{iCa}_{\mathrm{MB}}$, intradialytic $\mathrm{Ca}$ buffer capacity, $\Delta \mathrm{iCa}$, and $\Delta \mathrm{tCa}$ ) and its association with $\mathrm{CV}$ disease in 40 chronic $\mathrm{HD}$ patients. We demonstrate that $\mathrm{iCa}_{\mathrm{MB}}$ is independently determined by the dialysate-to-blood gradient at HD start and provide robust evidence that a rapidly accessible and exchangeable Ca pool exists in vivo that acutely counteracts serum $\mathrm{Ca}$ deviations in HD patients in the event of high intradialytic $\mathrm{Ca}_{\mathrm{MB}}$ : using $3.5 \mathrm{mEq} / \mathrm{L} \mathrm{dCa}$, the mean intradialytic Ca buffer capacity was found to be $73( \pm 18) \%$, which is strikingly similar to a mathematical modeling approach by Gotch et al. demonstrating that approximately $76 \%$ of positive $\mathrm{Ca}_{\mathrm{MB}}$ is acutely buffered in chronic $\mathrm{HD}$ patients $[3,16]$ and analog to the observed $78 \%$ in our previous proof-of-concept study [4]. In line with these findings, the use of a novel single pool variable-volume calcium kinetics model proposed by di Filippo et al. $[8,17]$ suggested the existence of intradialytic calcium fluxes from the ECF compartment to a non-modeled pool. By demonstrating an independent positive association of $\triangle \mathrm{iCa}$ with the presence of $\mathrm{CHD}$ in chronic HD patients, we present novel evidence for the clinical relevance of intradialytic Ca kinetics assessment in vivo. Importantly, a significant negative correlation was found between $\Delta \mathrm{iCa}$ and intradialytic Ca buffer capacity $(p<0.01)$ in our study. So far, evidence for a potential role of $\Delta \mathrm{Ca}$ as $\mathrm{CV}$ risk factor is rather limited and solely based on $\Delta \mathrm{tCa}$ data, which has been previously demonstrated to be associated with aortic calcification [11] and statistically related to the increased risk for MI with the use of higher dCa among diabetic HD patients [12]. Although it was previously suggested that serum Ca kinetics assessment should be rather based on iCa than on tCa levels [18], only a single $\mathrm{Ca}_{\mathrm{MB}}$ study previously reported both total and ionized serum calcium data when evaluating the use of $2.7 \mathrm{mEq} / \mathrm{L}$ 
$\mathrm{dCa}$ [19]. In the present study, we measured both serum ionized and total $\Delta \mathrm{Ca}$ and found that the presence of $\mathrm{CHD}$ is independently associated with $\Delta \mathrm{iCa}$ but not $\Delta \mathrm{tCa}$. In contrast to the study by Tagawa et al. [12], our results are not limited to diabetic HD patients and based on an established dialysate-sided $\mathrm{iCa}_{\mathrm{MB}}$ assessment approach $[4,20]$. Unlike previous $\mathrm{Ca}_{\mathrm{MB}}$ studies, we measured both pre- and postfilter ionized $\mathrm{dCa}$ in order to account for known differences between prescribed label value prefilter $\mathrm{dCa}$ versus measured prefilter $\mathrm{dCa}$ [4]. Indeed, the mean ionized prefilter $\mathrm{dCa}$ was found to be $2.92( \pm 0.12)$ $\mathrm{mEq} / \mathrm{L}$, that is, $83 \%$ of $3.5 \mathrm{mEq} / \mathrm{L}$ dCa label value (Table 1). As the dialysate-to-blood iCa gradient is the main driving force of $\mathrm{iCa}_{\mathrm{MB}}$, the use of label value prefilter $\mathrm{dCa}$ for $\mathrm{iCa}_{\mathrm{MB}}$ calculation would result in a substantial overestimation of the latter. We, thus, accounted for the observed discrepancy between label value prefilter $\mathrm{dCa}$ and measured prefilter $\mathrm{dCa}$ that most likely results from $\mathrm{Ca}$ complexation with anions, for example, bicarbonate, in the dialysate fluid. Interestingly, no relevant differences between ionized and total dCa-based $\mathrm{Ca}_{\mathrm{MB}}$ assessment have been observed in previous studies [5, 21]. Without assessing $\mathrm{Ca}_{\mathrm{MB}}$, however, previous studies focussing on $\Delta \mathrm{Ca}$ could not answer the question whether high intradialytic Ca load or altered extracellular Ca buffering per se affects $\mathrm{CV}$ risk. Importantly, $\mathrm{iCa}_{\mathrm{MB}}$ did not correlate with intradialytic $\mathrm{Ca}$ buffer capacity, $\Delta \mathrm{iCa}, \Delta \mathrm{tCa}$, or $\triangle \mathrm{iCa}_{\mathrm{ECF}}$ in our study, suggesting that intradialytic $\mathrm{Ca}$ burden - an established CV risk factor [22] - and Ca buffering are distinct processes that should be considered as independent $\mathrm{CV}$ risk factors. Based on a 2.2-fold increase in serum tCa compared with serum iCa, we approximatively calculated that $30 \%$ of buffered $\mathrm{iCa}_{\mathrm{MB}}$ is actually protein-bound; however, the remaining $70 \%$ are buffered outside the ECF compartment. In this regard, the involvement of osteocalcin, the most abundant noncollagenous bone protein $[23,24]$, in the rapidly accessible and exchangeable Ca pool has been suggested previously [25] and first experimentally described in vivo by our workgroup [4]. In this regard, osteocalcin has been supposed to stabilize bone surface brushite layer, which might act as rapidly accessible and exchangeable Ca pool [10]. By confirming the positive independent association of ucOC, that is, a circulating form of OC that does not undergo carboxylation or is actively decarboxylated [26, 27], with intradialytic Ca buffer capacity in the present study, we further support the hypothesis of bone being - at least partly - involved in acute extracellular Ca regulation [10]. It is tempting to speculate that extraosseous Ca storage, for example, in the vasculature and soft tissues, acts syn- ergistically in terms of intradialytic Ca buffering, but - in contrast to rapid osseous Ca storage - rather increases CV risk. In this context, we found a negative correlation of cOC levels with PWV, an established marker of vascular calcification and CV disease in HD patients [28], thereby indicating a potential shift from bone to vascular Ca storage: cOC is synthesized by mature osteoblasts, stimulated by active vitamin $\mathrm{D}$, and constitutively present in bone matrix; however, after carboxylation, a proportion can effuse in the circulation [24]. In this regard, we found a positive correlation of active vitamin $\mathrm{D}$ dose with ucOC $(p<0.01)$ and $\mathrm{cOC}(\mathrm{ns})$ in our study cohort. Assuming its role as a brushite stabilizer, reduced $\mathrm{cOC}$ levels might indicate reduced bone $\mathrm{Ca}$ buffer capacity and higher $\mathrm{CV}$ risk due to compensatory extraosseous Ca deposition, which is reflected by increased PWV (but not necessarily by reduced overall Ca buffer capacity). This is in line with several in vivo studies demonstrating an inverse relationship between circulating osteocalcin levels and CV risk both in the general [29-33] and HD population, where osteocalcin was suggested to have a vasculoprotective role [34-37]. While a few studies found contradictory results [38-40], recent evidence even suggested a U-shaped relationship [41, 42]. Animal studies also revealed conflicting results, with some demonstrating an inhibitory effect of osteocalcin on bone formation [43] and others identifying osteocalcin as potential promotor of osteochondrogenic differentiation of vascular smooth muscle cells during vitamin D-induced vascular calcification [44]. Growing evidence now suggests a dual role of osteocalcin, with different circulating osteocalcin isoforms that regulate vascular calcification and act as endocrine mediator, for example, by regulating insulin secretion and sensitivity, as well as tissue-expressed osteocalcin directly affecting the calcification process [27, 37]. Likewise, our study provides in vivo evidence for a predictive potential of different circulating osteocalcin isoforms in HD patients, that is, ucOC for intradialytic Ca buffering and cOC for a shift from bone to vascular calcium storage. Based on the strong independent correlation of aortic PWV with age, a much larger HD cohort would be statistically required to elucidate an independent association of cOC with elevated PWV. Our present study validates previous study findings from smaller HD cohorts [4] in an independent larger patient population and presents novel evidence for the clinical relevance of assessing intradialytic calcium kinetics by showing an independent association of $\Delta \mathrm{iCa}$ with CHD. Our data also support the potential role of OC as a marker of altered intradialytic Ca buffer capacity and vascular calcification thereby reflecting the complex in- 
terplay between bone- and vascular Ca storage in $\mathrm{HD}$ patients. Overall, our results strengthen the concept of a rapidly accessible and exchangeable $\mathrm{Ca}$ pool involved in intradialytic Ca regulation in vivo. Cross-sectional study design and the small patient number are main study limitations. Thus, prospective studies in larger HD patient cohorts are needed to evaluate the prognostic potential of intradialytic $\mathrm{Ca}$ kinetics parameters.

\section{Acknowledgement}

We thank the patients for study participation, the dialysis staff for supporting the study, and Dr. Herbert Schramek for his valuable contribution during manuscript preparation.

\section{Statement of Ethics}

This study was conducted in accordance with the World Medical Association Declaration of Helsinki. Written informed consent was obtained from each study participant prior to study inclusion using a standardized patient information and consensus form according to Good Clinical Practice (GCP) guidelines, and the study protocol was approved by the Innsbruck Medical University ethics committee prior to study initiation (protocol number AN2014-0313 343/4.7 391/5.3). All patient-associated information was managed entirely coded. Data collection was conducted using a standardized evaluation form (case report form) according to GCP recommendations. All patient-associated samples and clinical data are subject to privacy protection according to the current European General Data Protection Regulation. This study complies with the STROBE standards for reporting of observational studies.

\section{Conflict of Interest Statement}

The authors have no conflicts of interest to declare.

\section{Funding Sources}

The Austrian National Bank supported this work by providing an educational research grant (OENB Jubiläumsfonds Project No. 15366) to M.P. The funders did not have any role in study design; collection, analysis, and interpretation of data; writing the report; and the decision to submit the report for publication.

\section{Author Contributions}

M.P.: research idea and study design; L.F. and R.H.: data acquisition; M.P., L.F., R.H., and T.R.: data analysis/interpretation; M.P. and T.R.: statistical analysis; M.P. and G.M.: manuscript preparation and drafting; M.P., L.F., R.H., T.R., and G.M: approval of the final version.

\section{References}

1 Kidney Disease: Improving Global Outcomes CKDMBDWG. KDIGO clinical practice guideline for the diagnosis, evaluation, prevention, and treatment of chronic kidney disease-mMineral and bone disorder (CKDMBD). Kidney Int Suppl. 2009 Aug;73(113): S1-130.

2 van der Sande FM, ter Meulen KJA, Kotanko P, Kooman JP. Dialysate calcium levels: do they matter? Blood Purif. 2018 Dec:47(3): 230-5.

3 Gotch FA, Kotanko P, Thijssen S, Levin NW. The KDIGO guideline for dialysate calcium will result in an increased incidence of calcium accumulation in hemodialysis patients. Kidney Int. 2010 Aug;78(4):343-50.

4 Pirklbauer M, Schupart R, Mayer G. Acute calcium kinetics in haemodialysis patients. Eur J Clin Invest. 2016 Dec;46(12):976-84.

5 Basile C, Libutti P, Di Turo AL, Tundo S, Maselli $\mathrm{P}$, Casucci F, et al. Calcium mass balances during standard bicarbonate hemodialysis and long-hour slow-flow bicarbonate hemodialysis. J Nephrol. 2011;24(6):742, 8.

6 Maheshwari V, Cherif A, Fuertinger D, Schappacher-Tilp G, Preciado P, Thijssen S, et al. An in silico method to predict net calcium trans- fer during hemodialysis. Conf Proc IEEE Eng Med Biol Soc. 2017 Jul;2017:2740-3.

7 Di Filippo S, Bellasi A, Locatelli F. Serum calcium may not accurately predict intradialytic calcium mass transfer. Hemodial Int. 2016 Apr;20(2):331-2.

8 di Filippo S, Carfagna F, la Milia V, Bellasi A, Casagrande G, Bianchi C, et al. Assessment of intradialysis calcium mass balance by a single pool variable-volume calcium kinetic model. Hemodial Int. 2018;22(1):126-35.

9 Byrne FN, Kinsella S, Murnaghan DJ, Kiely M, Eustace JA. Lack of correlation between calcium intake and serum calcium levels in stable haemodialysis subjects. Nephron Clin Pract. 2009;113(3):c162-8.

10 Pirklbauer M, Mayer G. The exchangeable calcium pool: physiology and pathophysiology in chronic kidney disease. Nephrol Dial Transplant. 2011 Aug;26(8):2438-44.

11 Yamada K, Fujimoto S, Nishiura R, Komatsu H, Tatsumoto M, Sato Y, et al. Risk factors of the progression of abdominal aortic calcification in patients on chronic haemodialysis. Nephrol Dial Transplant. 2007 Jul;22(7):2032-7.

12 Tagawa M, Hamano T, Sueta S, Ogata S, Saito Y. Higher dialysate calcium concentration is associated with incident myocardial infarction among diabetic patients with low bone turnover: a longitudinal study. Sci Rep. 2018 Jul;8(1):10060.

13 Bosticardo G, Malberti F, Basile C, Leardini L, Libutti P, Filiberti O, et al. Optimizing the dialysate calcium concentration in bicarbonate haemodialysis. Nephrol Dial Transplant. 2012 Jun;27(6):2489-96.

14 Fogh-Andersen N, Altura BM, Altura BT, Siggaard-Andersen O. Composition of interstitial fluid. Clin Chem. 1995 Oct;41(10): 1522-5.

15 Wassertheurer S, Kropf J, Weber T, van der Giet M, Baulmann J, Ammer M, et al. A new oscillometric method for pulse wave analysis: comparison with a common tonometric method. J Hum Hypertens. 2010;24(8):498504 .

16 Gotch F, Kotanko P, Handelman G, Levin N. A kinetic model of calcium mass balance during dialysis therapy. Blood Purif. 2007;25(1): 139-49.

17 Carfagna F, Di Filippo S, Bellasi A, Pontoriero G, Locatelli F. Shed a light on intradialytic calcium mass balance. Kidney Int. 2016 Jun; 89(6):1402.
Calcium Kinetics and Cardiovascular Disease in Hemodialysis Patients 
18 Gouri A, Dekaken A. A comparison of corrected serum calcium levels to ionized calcium levels in haemodialysis patients. Ann Biol Clin. 2012 Mar-Apr;70(2):210-2.

19 Waniewski J, Debowska M, Wojcik-Zaluska A, Ksiazek A, Zaluska W. Quantification of dialytic removal and extracellular calcium mass balance during a weekly cycle of hemodialysis. PloS One. 2016;11(4):e0153285.

20 Basile C, Libutti P, Di Turo AL, Vernaglione L, Casucci F, Losurdo N, et al. Effect of dialysate calcium concentrations on parathyroid hormone and calcium balance during a single dialysis session using bicarbonate hemodialysis: a crossover clinical trial. Am J Kidney Dis. 2012 Jan;59(1):92-101.

21 Argilés A, Mion CM. Calcium balance and intact PTH variations during haemodiafiltration. Nephrol Dial Transplant. 1995 Nov; 10(11):2083-9.

22 Goodman WG, Goldin J, Kuizon BD, Yoon C, Gales B, Sider D, et al. Coronary-artery calcification in young adults with end-stage renal disease who are undergoing dialysis. N Engl J Med. 2000 May 18;342(20):1478-83.

23 Hauschka PV, Lian JB, Cole DE, Gundberg CM. Osteocalcin and matrix Gla protein: vitamin K-dependent proteins in bone. Physiol Rev. 1989 Jul;69(3):990-1047.

24 Kerner SA, Scott RA, Pike JW. Sequence elements in the human osteocalcin gene confer basal activation and inducible response to hormonal vitamin D3. Proc Natl Acad Sci U S A. 1989 Jun;86(12):4455-9.

25 Talmage RV, Mobley HT. The concentration of free calcium in plasma is set by the extracellular action of noncollagenous proteins and hydroxyapatite. Gen Comp Endocrinol. 2009 Jul;162(3):245-50.

26 Lee AJ, Hodges S, Eastell R. Measurement of osteocalcin. Ann Clin Biochem. 2000 Jul; 37(Pt 4):432-46.

27 Li J, Zhang H, Yang C, Li Y, Dai Z. An overview of osteocalcin progress. J Bone Miner Metab. 2016 Jan 8;34(4):367-79.
28 Sarafidis PA, Loutradis C, Karpetas A, Tzanis G, Piperidou A, Koutroumpas G, et al. Ambulatory pulse wave velocity is a stronger predictor of cardiovascular events and all-cause mortality than office and ambulatory blood pressure in hemodialysis patients. Hypertension. 2017 Jul;70(1):148-57.

29 Confavreux CB, Szulc P, Casey R, Boutroy S, Varennes A, Vilayphiou N, et al. Higher serum osteocalcin is associated with lower abdominal aortic calcification progression and longer 10-year survival in elderly men of the MINOS cohort. J Clin Endocrinol Metab. 2013 Mar;98(3):1084-92.

30 Ogawa-Furuya N, Yamaguchi T, Yamamoto M, Kanazawa I, Sugimoto T. Serum osteocalcin levels are inversely associated with abdominal aortic calcification in men with type 2 diabetes mellitus. Osteoporos Int. 2013 Aug; 24(8):2223-30.

31 Yang R, Ma X, Dou J, Wang F, Luo Y, Li D, et al. Relationship between serum osteocalcin levels and carotid intima-media thickness in Chinese postmenopausal women. Menopause. 2013 Nov;20(11):1194-9.

32 Holvik K, van Schoor NM, Eekhoff EM, den Heijer M, Deeg DJ, Lips P, et al. Plasma osteocalcin levels as a predictor of cardiovascular disease in older men and women: a population-based cohort study. Eur J Endocrinol. 2014 Aug; 171(2):161-70

$33 \mathrm{Ma} \mathrm{H}$, Lin H, Hu Y, Li X, He W, Jin X, et al. Serum levels of osteocalcin in relation to glucose metabolism and carotid atherosclerosis in Chinese middle-aged and elderly male adults: the Shanghai Changfeng Study. Eur J Intern Med. 2014 Mar;25(3):259-64.

34 Lenora J, Ivaska KK, Obrant KJ, Gerdhem P. Prediction of bone loss using biochemical markers of bone turnover. Osteoporos Int. 2007 Sep;18(9):1297-305.

35 Osorio A, Ortega E, Torres JM, Sanchez P, Ruiz-Requena E. Mineral-bone metabolism markers in young hemodialysis patients. Clin Biochem. 2011 Dec;44(17-18):1425-8.

36 Osorio A, Ortega E, Torres JM, Sanchez P, Ruiz-Requena E. Biochemical markers of vascular calcification in elderly hemodialysis patients. Mol Cell Biochem. 2013 Feb;374(1-2): 21-7.
37 Csiky B, Sági B, Peti A, Lakatos O, Prémusz V, Sulyok E. The impact of osteocalcin, osteoprotegerin and osteopontin on arterial stiffness in chronic renal failure patients on hemodialysis. Kidney Blood Press Res. 2017; 42(6):1312-21.

38 Reyes-Garcia R, Rozas-Moreno P, JimenezMoleon JJ, Villoslada MJ, Garcia-Salcedo JA, Santana-Morales S, et al. Relationship between serum levels of osteocalcin and atherosclerotic disease in type 2 diabetes. Diabetes Metabol. 2012 Feb;38(1):76-81.

39 Choi BH, Joo NS, Kim MJ, Kim KM, Park KC, Kim YS. Coronary artery calcification is associated with high serum concentration of undercarboxylated osteocalcin in asymptomatic Korean men. Clin Endocrinol. 2015 Sep; 83(3):320-6.

40 Chi PJ, Lin YL, Tasi JP, Wang CH, Hou JS, Lee CJ, et al. Osteocalcin and carotid-femoral pulse wave velocity in patients on peritoneal dialysis. Tzu Chi Med J. 2019 Jan-Mar;31(1): 23-8.

41 Yeap BB, Chubb SA, Flicker L, McCaul KA, Ebeling PR, Hankey GJ, et al. Associations of total osteocalcin with all-cause and cardiovascular mortality in older men. The Health In Men Study. Osteoporos Int. 2012 Feb;23(2): 599-606.

42 Lerchbaum E, Schwetz V, Pilz S, Grammer TB, Look M, Boehm BO, et al. Association of bone turnover markers with mortality in men referred to coronary angiography. Osteoporos Int. 2013 Apr;24(4):1321-32.

43 Ducy P, Desbois C, Boyce B, Pinero G, Story B, Dunstan C, et al. Increased bone formation in osteocalcin-deficient mice. Nature. 1996 Aug 1;382(6590):448-52.

44 Idelevich A, Rais Y, Monsonego-Ornan E. Bone Gla protein increases HIF-1alpha-dependent glucose metabolism and induces cartilage and vascular calcification. Arterioscler Thromb Vasc Biol. 2011 Sep;31(9):e55-71. 